\title{
Electronic Properties of Twisted Bilayer Graphene in the Presence of a Magnetic Field
}

\author{
M. F. C. Martins Quintela ${ }^{1, *}, J . C . C$. Guerra ${ }^{1, * *}$, and S. M. João ${ }^{1, * * *}$ \\ ${ }^{1}$ Centro de Física das Universidades do Minho e do Porto, CF-UM-UP Departmento de Física e Astrono- \\ mia, Universidade do Porto, Rua do Campo Alegre 4169-007, Porto, Portugal
}

\begin{abstract}
In AA-stacked twisted bilayer graphene, the lower energy bands become completely flat when the twist angle passes through certain specific values: the socalled "magic angles". The Dirac peak appears at zero energy due to the flattening of these bands when the twist angle is sufficiently small [1-3]. When a constant perpendicular magnetic field is applied, Landau levels start appearing as expected [5]. We used the Kernel Polynomial Method (KPM) [6] as implemented in KITE [7] to study the optical and electronic properties of these systems. The aim of this work is to analyze how the features of these quantities change with the twist angle in the presence of an uniform magnetic field.
\end{abstract}

\section{Introduction}

Until very recently, the origin of the magic angles, which are characterized by the extreme flattening of the lowest energy bands, in twisted bilayer graphene (TBG) was unknown. In 2007 and 2012, J. Lopes dos Santos, N. Peres et al published two articles [1,2] where they developed a continuum model for this material. With this work, they were able to find a simple relation between supperlattice vectors with which they could explain the physics of all types of commensurate structures. Very recently, in 2019, A. Vishwanath went over the origin for these magic angles and their periodicity in $\alpha \sim 1 / \theta$ [3].

Using KITE, which employs a Chebyshev polynomial-approach to approximate Green's functions, it is possible to calculate the density of states for a multitude of $2 \mathrm{D}$ and $3 \mathrm{D}$ structures. This allows us to calculate the density of states for several commensurable angles, as well as the optical conductivity and the local density of states [7].

\subsection{Moiré Superlattice and Hamiltonian}

While the structure of graphene, the honeycomb lattice, is reasonably simple, it becomes quite complex when two graphene sheets are stacked and a relative twist is applied to one of them. For some angles (denominated commensurate angles), a periodic structure emerges with a periodicity much larger than that of the individual graphene lattices. This is the Moire superlattice, which is visible in Figure 1 with central AA-stacking and a twist angle of 21.798 degrees.

\footnotetext{
*e-mail: mfcmquintela@fc.up.pt

**e-mail: cguerrajoao@gmail.com

***e-mail: up201206827@fc.up.pt
} 


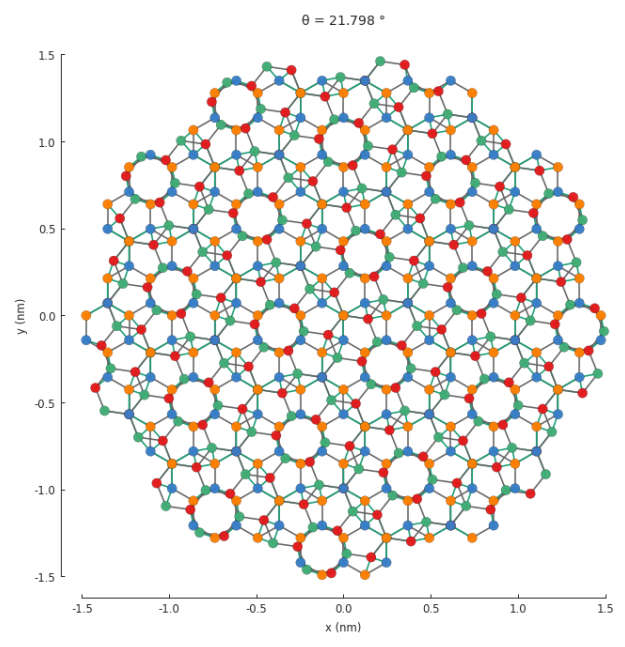

Figure 1. Moiré superlattice for a twist angle of 21.798 degrees

The second quantization tight-binding Hamiltonian [4] can be written as a sum of an intralayer term and an inter-layer one

$$
\begin{aligned}
H & =H_{\|}+H_{\perp} \\
& =-\sum_{i \neq j, m} \gamma_{i j}^{m m}\left(c_{m, i}^{\dagger} c_{m, j}+\text { h.c. }\right)-\sum_{i, j, m} \gamma_{i j}^{m, m+1}\left(c_{m, i}^{\dagger} c_{m+1, j}+\text { h.c. }\right)
\end{aligned}
$$

where $c_{m, i}^{\dagger}, c_{m, i}$ are, respectively, the creation and annihilation operators of a $p_{z}$ state in the atomic site $i$ in layer $m$, and $\gamma_{i j}^{m m}$ is the in-plane hopping integral between these atomic sites. As we are only considering a nearest neighbour intra-layer hopping, $\gamma_{i j}^{m m}$ will be zero when the atomic sites are not first-neighbours, and, when they are, will be given by $V_{p p \pi}^{0}$ (the nearest neighbours hopping parameter for single-layer graphene, obtained via DFT). For the inter-layer term, we are considering the hopping integral to be of the form

$$
t(r)=V_{p p \sigma}^{0}\left(1-\frac{c_{0}^{2}}{r^{2}}\right) e^{-\frac{r-c_{0}}{\delta}}
$$

where $r$ is the $3 \mathrm{D}$ distance between the two atomic sites, $c_{0}$ is the inter-layer distance, $V_{p p \sigma}^{0}$ is the hopping integral between two vertically-stacked atoms in untwisted bilayer graphene, and $\delta$ is a parameter that modulates the hopping cutoff at long distances (also obtained via DFT).

\section{Twisted Bilayer Graphene in a Magnetic Field}

Due to the effect of a perpendicular magnetic field in monolayer graphene [5], its energy spectrum becomes quantized into Landau Levels, whose expression is given by

$$
\epsilon_{n}=\operatorname{sign}(n) \frac{\hbar v_{F} \sqrt{2}}{l_{B}} \sqrt{|n|}
$$

where $v_{F}$ is the Fermi velocity, and the magnetic length $l_{B}$ is defined as

$$
l_{B}=\sqrt{\frac{\hbar}{e B}} .
$$


This also creates specific selection rules for the transitions between energy levels, which will modify its optical properties.

\subsection{Density of States and Landau Levels}

To study the dependence on the twist angle of the density of states, we first calculated it using KITE [7] for several commensurate angles without any magnetic field. In agreement with [8], the Dirac Peak, which is caused by the forming of a flat-band at zero energy, appears for sufficiently low twist angles (less than 3 degrees), around which two gaps open up. This is visible in Figure 2.

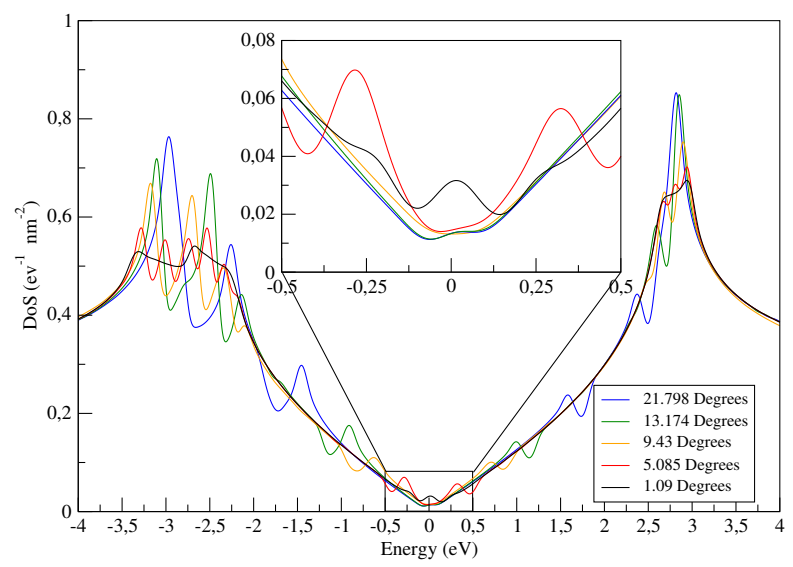

Figure 2. Density of states for several twist angles

When the magnetic field is added using the Peierls substitution [9]

$$
t\left(r_{j}-r_{i}\right) \rightarrow \tilde{t}\left(r_{j}-r_{i}\right)=t\left(r_{j}-r_{i}\right) e^{-i \frac{e}{\hbar} \int_{r_{i}}^{r_{j}} \mathbf{A}\left(r^{\prime}\right) \cdot d \mathbf{r}^{\prime}}
$$

the energy levels change qualitatively. The crossing of each Landau Level creates well-known peaks in the density of states [11]. To analyze the differences, the densities of states for both $B=0 \mathrm{~T}$ and for $B=24 \mathrm{~T}$ are plotted together for four different twist angles.
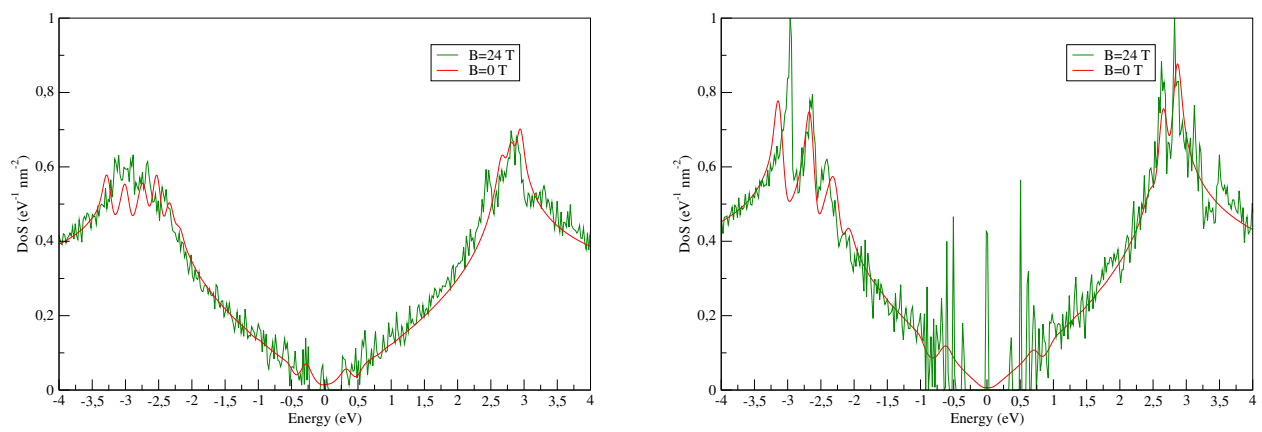

Figure 3. Comparison of the densities of states for a twist angle of 5.085 and 9.430 degrees 

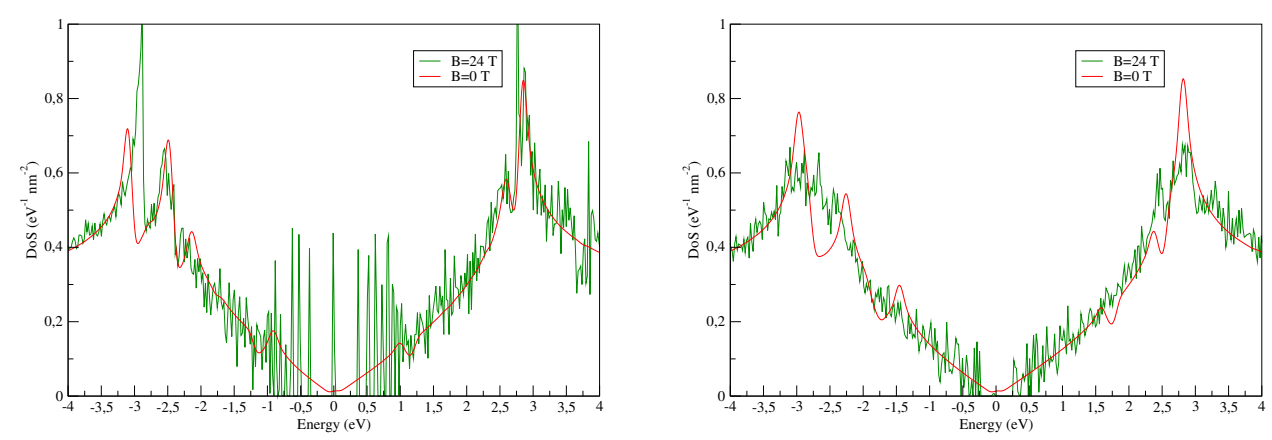

Figure 4. Comparison of the densities of states for a twist angle of 13.174 and 21.798 degrees

The above-mentioned peaks due to the crossing of the LLs are more clearly visible for 9.43 and 13.174 degrees. Because of this, we will use one of these angles for our study.

\subsection{Conductivity}

To finalize this proceeding, we will take a look at the DC conductivity in both the $x x$ and the $x y$ directions, which were obtained through KITE. For this purpose, KITE employs the Kubo-Bastin formula in the velocity gauge:

$$
\sigma_{\mathrm{DC}}^{\alpha \beta}=\frac{-e^{2}}{V} \int_{-\infty}^{\infty} d \epsilon f(\epsilon) \operatorname{Tr}\left[\delta(\epsilon-\hat{H}) \hat{v}^{\alpha} \frac{d G^{+}}{d \epsilon}(\epsilon) \hat{v}^{\beta}-\delta(\epsilon-\hat{H}) \hat{v}^{\beta} \frac{d G^{-}}{d \epsilon}(\epsilon) \hat{v}^{\alpha}\right]
$$

where $G^{ \pm}$are the retarded/advanced Green's functions respectively, defined by

$$
G^{ \pm}(\epsilon)=\frac{1}{\epsilon-\hat{H} \pm i 0^{+}} .
$$

By construction of this formula, the imaginary part of the DC-conductivity will be zero. As such, we will focus only on the real part of both $\sigma_{\mathrm{DC}}^{x x}$ and $\sigma_{\mathrm{DC}}^{x y}$, which we plot for a twist angle of 9.43 degrees and a magnetic field of $24 \mathrm{~T}$ in Figure 5.
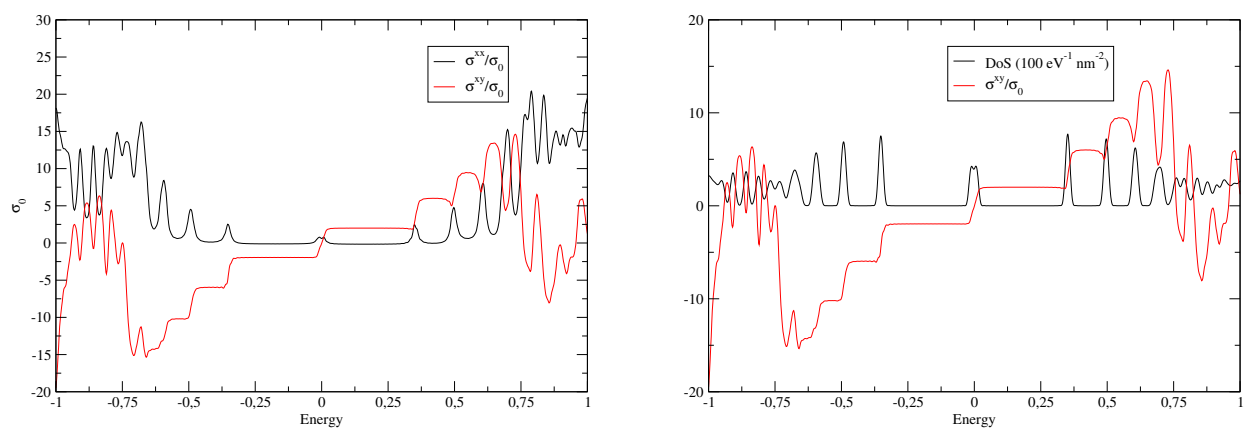

Figure 5. (Left) Real part of $\sigma_{\mathrm{DC}}^{x x}$ and $\sigma_{\mathrm{DC}}^{x y}$

(Right) Real part of $\sigma_{\mathrm{DC}}^{x y}$ juxtaposed onto the (rescaled) Density of States

The qualitative behaviour of these quantities is as expected, with their peaks and plateaus upon the crossing of each Landau Level (visible by the sudden spike in the density of states). 


\section{Conclusions and Future Work}

Twisted bilayer graphene is a very promising material, with a vast array of useful properties, amongst which is the flattening of the energy bands due to the vanishing of the Fermi velocity, which leads to superconducting states [11]. We were able to obtain the DC conductivity in both the $x x$ and the $x y$ directions in the presence of a magnetic field through KITE. In the future, we would want to study the angle dependence of these quantities, as was done previously for both the presence of disorder and/or a perpendicular electric field [12]. One point that will be of interest for future study is doing this same comparison for optical conductivity [13], both first and second order.

\section{Acknowledgements}

The authors acknowledge financing of Fundação da Ciência e Tecnologia, of COM-PETE 2020 program in FEDER component (European Union), through projects POCI-01-0145-FEDER-028887 and UID/FIS/04650/2013. The authors also acknowledge financial support from Fundação para a Ciência e Tecnologia, Portugal, through national funds, co-financed by COMPETE-FEDER (grant M-

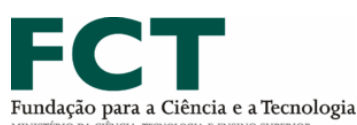
ERA- NET2/0002/2016 - UltraGraf) under the Partnership Agreement PT2020.

\section{References}

[1] Lopes dos Santos, J., Peres, N., Castro Neto, A., Physical Review B 86, 155449 (2012)

[2] Lopes dos Santos, J., Peres, N., Castro Neto, A., Physical Review Letters 99, 256802 (2007)

[3] Vishwanath, A., Kruchkov, A., Tarnopolsky, G., Physical Review Letters 122, 106405 (2019)

[4] Moon, P., Koshino, M., Phys. Rev. B 85, 195458 (2012)

[5] Goerbig, M. O., Reviews of Modern Physics 83, 1193 (2011)

[6] Weiße, A., Wellein, G., Alvermann, A., Reviews of Modern Physics 78, 275 (2016)

[7] Lopes, J.V., João, S.M., Ferreira, A., Covaci, L., Andelkovic, M., Rappoport. T., (2018) Quantum KITE. Website: https://quantum-kite.com/

[8] Moon, P., Koshino, M., Physical Review B 85, 195458 (2012)

[9] Peierls, R., Z. Phys. 80: 763-791 (1933)

[10] Anh Le, H., Nam Do, V., Physical Review B 97, 125136 (2018)

[11] Cao, Y., Fatemi, V., Fang, S., et al, Nature 556, 43 (2018)

[12] Anđelković, M., Covaci, L., Peeters, F. M., Phys. Rev. Materials 2, 034004 (2018)

[13] Catarina, G., Amorim, B., Castro, E. V., Lopes, J. V., Peres, N., Handbook of Graphene: Volume 3, Chap. 6, pp. 177-230 (2019) 